\title{
MiR-146a mediates TLR-4 signaling pathway to affect myocardial fibrosis in rat constrictive pericarditis model
}

\author{
Yangjie Xiao, Wei Qiao, Xin Wang, Lijuan Sun, Weidong Ren \\ Department of Ultrasound, Shengjing Hospital of China Medical University, Shenyang, China \\ Contributions: (I) Conception and design: Y Xiao, W Ren; (II) Administrative support: W Ren; (III) Provision of study materials: Y Xiao, W Qiao; (IV) \\ Collection and assembly of data: Y Xiao, W Qiao; (V) Data analysis and interpretation: X Wang, L Sun; (VI) Manuscript writing: All authors; (VII) \\ Final approval of manuscript: All authors. \\ Correspondence to: Weidong Ren, MD, PhD. Department of Ultrasound, Shengjing Hospital of China Medical University, 36 Sanhao St, Heping \\ District, Shenyang 110004, China. Email: renwdcmu@163.com.
}

Background: Myocardial fibrosis (MF) is thought to be associated with constrictive pericarditis (CP). miR146a has been reported to be related to the survival of myocardial fibroblasts and related signal transduction pathways. The aim of this study was to investigate the expression of miR-146a in CP with MF and the activation of the Toll-like receptor 4 (TLR-4) signaling pathway, to understand the molecular mechanism of MF involvement in CP.

Methods: Thirty rats with different disease duration were randomly divided into three groups: an 8-week model group (CP-8W group), a 16-week model group (CP-16W group) model, and a normal control group (N group). After the CP model was established in the rats, the myocardial tissues were collected. The expression of miR-146a, the key factors of TLR-4 signaling pathway, including IL-1 receptor-associated kinase 1 (IRAK1), tumor necrosis factor receptor-associated factor 6 (TRAF6), nuclear factor- $\kappa \mathrm{B}$ (NF$\kappa \mathrm{B})$ and $\mathrm{p}-\mathrm{NF}-\kappa \mathrm{B}$, and the MF indicator $\alpha-\mathrm{SMA}$ in myocardial tissue were detected. After treatment with lipopolysaccharide (LPS), primary cultured rat cardiac fibroblasts (CFs) were transfected with miR-146a. RT-PCR and western blot were used to detect the expression of downstream effectors to further verify the function of miRNA-146a in regulating MF via the TLR-4 signaling pathway.

Results: miR-146a was increased in the CP-8W group but not in the CP-16W group. IRAK1 and TRAF6 in the CP-16W group were found to be higher than in the $\mathrm{N}$ group and CP- $8 \mathrm{~W}$ group. $\alpha$-SMA in the model groups was higher than in the $\mathrm{N}$ group. Compared with the CP-8W group, $\alpha$-SMA in the CP-16W model group was further increased. In the experiments using CFs, the expression of IRAK1, TRAF6, p-NF- $\mathrm{B}$ and $\alpha$-SMA increased in the LPS-treated group compared with the $\mathrm{N}$ group. After transfection of CFs with the miR-146a mimics, the expression of IRAK1, TRAF6, p-NF- $\kappa$ B and $\alpha$-SMA decreased compared with the LPS-treated group. Following transfection of CFs with miR-146a inhibitors, the expression of IRAK1, TRAF6, p-NF- $\kappa$ B and $\alpha$-SMA increased compared with the LPS-treated group.

Conclusions: The expression of miR-146a demonstrated a dynamic change in the CP model; it was increased at the early time point $(\mathrm{CP}-8 \mathrm{~W})$ and then decreased at the $16 \mathrm{~W}$ time point. miR-146a suppressed MF by inhibiting the target genes TRAF6 and IRAK1 via the TLR-4 signaling pathway.

Keywords: Constrictive pericarditis (CP); myocardial fibrosis (MF); miR-146a; IL-1 receptor-associated kinase 1 (IRAK1); tumor necrosis factor receptor-associated factor 6 (TNFR6)

Submitted Aug 21, 2020. Accepted for publication Dec 13, 2020.

doi: $10.21037 /$ jtd-20-2716

View this article at: http://dx.doi.org/10.21037/jtd-20-2716 


\section{Introduction}

Constrictive pericarditis (CP) is a series of venous reflux disorder syndromes caused by chronic inflammation, which leads to pericardial thickening, calcification and adhesions, and results in limited left ventricular diastolic filling. The limited ventricular diastolic filling is due to the loss of elasticity of the pericardium. Surgical removal of the pericardium is the main treatment for $\mathrm{CP}$, but some patients have unsatisfactory recovery of myocardial function after surgery $(1,2)$, which may be related to myocardial fibrosis (MF) caused by pericarditis $(3,4)$. Therefore, it is of great value to identify targets for early intervention for $\mathrm{MF}$ in $\mathrm{CP}$.

Studies have reported that multiple miRNAs are involved in cardiac fibroblast (CF) survival and related signaling pathways. Some miRNAs directly target the genes coding for extracellular matrix proteins (5), while other miRNAs prevent CFs from acquiring an activated secretory phenotype (6). With the development of biological sequencing technology, several new miRNAs have been discovered, and a number of studies have been conducted on the relationship between abnormalities miRNA expression and MF. miR-146a is primarily involved in innate immune regulation, tumorigenesis and inflammatory development (7). Studies have shown that miR-146a plays an important role in tissue fibrosis, including liver and kidney fibrosis $(8,9)$. The role of miR146a in the MF process of CP is unclear, but has potential as a therapeutic target.

Toll-like receptors (TLRs) are important protein molecules involved in non-specific immunity and can recognize molecules with conserved structures derived from microorganisms (10). The key factors in the TLR-4 signaling pathway include IL-1 receptor-associated kinase 1 (IRAK1), tumor necrosis factor receptor-associated factor 6 (TRAF6) and nuclear factor- $\mathrm{KB}(\mathrm{NF}-\kappa \mathrm{B})(11)$. miR-146a can induce the TLR-4 signaling pathway to participate in the disease process. It was found that miR-146a can inhibit low-density lipoprotein cholesterol accumulation and the cellular inflammatory response by regulating the TLR-4 pathway, and the expression of miR-146a is first increased and then decreased in atherosclerotic endothelial cells. The increased miR-146a has a therapeutic effect in atherosclerosis (12).

In our previous study, the rat was used as the model subject, and a method for CP was explored that utilized injection of an inflammatory agent into the pericardium without opening the thoracic compartment (13). Therefore, in this study, the role of miR-146a and the related TLR4 pathway was investigated in a rat model of CP involving
MF and cultured CFs. We present the following article in accordance with the ARRIVE reporting checklist (available at http://dx.doi.org/10.21037/jtd-20-2716).

\section{Methods}

\section{Development of rat CP model}

Models were constructed as previously reported (13). Thirty male Wistar rats purchased from Liaoning Changsheng Biotechnology Co., Ltd. were raised in the Animal Experimental Center of Shengjing Hospital. The rats were randomly divided into three groups (ten per group): a group of rats raised 8 weeks (CP-8W group) and 16 weeks (CP$16 \mathrm{~W}$ group) after injection of inflammation solution and an untreated normal group ( $\mathrm{N}$ group) as the control. All the rats were kept in pathogen-free housing under a $12 \mathrm{~h}$ light-dark cycle with free access to food and sterile water during the research procedures. Anesthesia was given by intraperitoneal injection of a $3 \%$ sodium pentobarbital solution at $50 \mathrm{mg} / \mathrm{kg}$. After successful anesthesia, the rat chest hair was shaved and a needle was inserted at 10-15 degrees left tilt into the chest wall close to the right edge of the sternum, three ribs from the xiphoid. The needle was advanced until a hollow feeling was identified. The depth of the needle was about $3-5 \mathrm{~mm}$. The model groups were injected with $1 \mathrm{ml}$ of inflammation solution [lipopolysaccharide (LPS, Sigma, MO, USA) solution and talcum powder (Sinopharm, Beijing, China) suspension were mixed at a ratio of 1:1] at the first week and the ninth week, and ejections were repeated the next week. Together with untreated normal rats ( $\mathrm{N}$ group), they were all fed to the 16th week. All animal experiments were approved by the Ethics Committee of Shengjing Hospital (2015PS152K). The treatment of the animals during the experiment complied with the Guidelines for Animal Ethics Treatment from the Ministry of Science and Technology.

\section{Pathology of the myocardium and pericardium}

The rats were sacrificed by cervical dislocation while anesthetized after 16 weeks later, after taking off the parietal pericardium and cutting off the great vessels and atrium tissues, the ventricles were cut into two sections from the middle. The apex sections were fixed in $4 \%$ paraformaldehyde (Sinopharm, Beijing, China) for staining. The bottom sections were placed in cryopreservation tubes and frozen in liquid nitrogen for RNA and Western blot analysis. The morphological changes of the pericardium and 
myocardial tissues were observed by hematoxylin and eosin (Solarbio, Beijing, China) staining. A microscope and image acquisition system (Nikon, Tokyo, Japan) were used to observe and take continuous photos around the heart. The formation of collagen fibers in each group of myocardial tissue was detected using Sirius (Solarbio, Beijing, China) red staining. The microscope and image acquisition system were used to observe and take photos.

\section{Quantitative real-time PCR}

Total RNA was isolated from fresh frozen myocardial tissue using RNAiso Plus (Takara, Dalian, China). RNA was reverse-transcribed using PrimeScrip RT Reagent Kit (Takara). Quantitative real-time PCR was performed using SYBR Premix Ex Tag II (Takara) in a Real-Time PCR System (ABI, 7500, CA, USA) with an annealing temperature at $60^{\circ} \mathrm{C}$. The fold change of relative expression of target genes was calculated using the $2^{-\Delta \Delta C \mathrm{t}}$ method. After $\log$-transformation of the data, relative mRNA expression of target gene including IRAK1, TRAF6 and a -SMA was shown in the histogram. The primers were synthesized by Takara. GAPDH served as a normalizing control. The sequences are as follows: miR-146a-5p: 5'-gcgTGAGA ACTGAATTCCATGGGTT-3'; miR-146a-3p: 5'-gc gcACCTGTGAAGTTCAGTTCTTT-3'; a-SMA-F: 5'-CTGAGCGTGGCTATTCCTTC-3'; a-SMA-R: 5'-AGAAGAGGAAGCAGCAGTGG-3'; IRAK1-F: 5'-CATGGCAGCCTCTGGTAGTG-3'; IRAK1-R: 5'-GGTAGCAGAGAGGCCAGGAA-3'; TRAF6-F: 5'-TCATGCACCTGGAAGCCTTA-3'; TRAF6-R: 5'-TCAGTACTCCGTGGCTGGAA-3'; GAPDH-F: 5 '-TGGGAAGCTGGTCATCAAC-3'; GAPDH-R: 5' - GCATCACCCCATTTGATGTT-3'. Three independent experiments were performed.

\section{Western blot analysis}

Fresh myocardial tissue was lysed in western blot lysis buffer containing $1 \mathrm{mM}$ phenylmethanesulfonylfluoride (Beyotime, Shanghai, China). Total proteins were extracted from the lysate and quantified using a bicinchoninic acid protein assay. Polyacrylamide gels (Beyotime) of different concentrations were used based on the molecular weight of proteins to be tested. After samples were loaded and electrophoresed, proteins were transferred to polyvinylidene fluoride membranes using the standard wet transfer method. After blocking with $5 \%$ non-fat milk for $2 \mathrm{~h}$, membranes were incubated with primary antibodies in TBST overnight at $4{ }^{\circ} \mathrm{C}$. The following primary antibodies were used: anti- $\alpha-$ SMA (1:10,000, Abcam, Cambridge, UK), IRAK1 (1:1,000, Proteintech, IL, USA), TRAF6 (1:1,000, Abcam), NF$\kappa \mathrm{B}(1: 1,000$, Abcam $), \mathrm{p}-\mathrm{NF}-\kappa \mathrm{B}(1: 1,000, \mathrm{Abcam}), \alpha$-SMA (1:1,000, Abcam) and GAPDH (1:10,000, Proteintech). After washing with TBST, membranes were incubated with a secondary antibody at $37^{\circ} \mathrm{C}$ for $2 \mathrm{~h}$. Immunoreactive bands were detected by enhanced chemiluminescence ECL (GE, MA, USA) and scanned in the Gel Doc XR system (Azure, C300, CA, USA). Images were analyzed with Image Lab software. Three independent experiments were performed. Averages of all three was analyzed.

\section{CFs extraction, culture and transfection}

CFs were taken from the heart of Wistar rats 24-36 h after birth. The cells were cultured in HG/DMEM (Life Technologies, CA, USA) medium containing 10\% FBS (Biological Industries, Kibbutz Beit Haemek, Israel) in a $37{ }^{\circ} \mathrm{C}, 5 \% \mathrm{CO}_{2}$ incubator. $\mathrm{CFs}\left(2 \times 10^{5}\right)$ were seeded and cultured in 6 -well cell culture plates until they reached approximately $70 \%$ confluency. Lipofectamine 3000 (Invitrogen, CA, USA) was used for transient transfections according to the manufacturer's protocol. miR-146a mimics and inhibitors were used. CFs from the experiment groups were cultured in HG/DMEM medium containing $7.5 \mu \mathrm{L}$ Lipofectamine 3000 and $7.5 \mu \mathrm{L}$ siRNA (miR-146a$5 \mathrm{p}$ mimics, miR-146a-5p inhibitors, negative control and negative control inhibitor, respectively) for $6 \mathrm{~h}$, then cultured in medium containing $10 \mu \mathrm{g} / \mathrm{mL}$ LPS for $48 \mathrm{~h}$ before harvested. CFs in the LPS group were treated only with LPS. CFs in the control group were treated without transfection or LPS. miRNA sequences were as follows: miR-146a5p mimics 5'-UGAGAACUGAAUUCCAUGGGUU-3'; miR-146a-5p inhibitors 5'-AACCCAUGGAAU UCAGUUCUCA-3'; Negative control 5'-UUCU CCGAACGUGUCACGUTT-3'; Negative control inhibitor 5'-CAGUACUUUUGUGUAGUACAA-3' . Three independent experiments were performed.

\section{Immunofluorescence}

Vimentin immunofluorescence was used to identity CFs. Cell slides were added to a 24 -well plate, and $2.0 \times 10^{4}$ cells per well were plated for $24 \mathrm{~h}$ (cell fusion rate was about $70 \%)$, and $30 \mu \mathrm{L}$ of Vimentin antibody (1:250, Proteintech) or $\alpha$-SMA (1:250, Abcam) antibody working solution was 
added to each slide. Slides were incubated overnight in a wet box at $4{ }^{\circ} \mathrm{C}(16 \mathrm{~h})$. After washing, $30 \mu \mathrm{L}$ of goat antimouse fluorescent secondary antibody working solution (1:1,000, Abcam) was added to each climbing piece and incubated at room temperature for $2 \mathrm{~h}$ in the dark. After washing, $30 \mu \mathrm{L}$ of 4',6-diamidino-2-phenylindole (DAPI) (Beyotime, Beijing, China) stain was added to each slide and stained at room temperature for $15 \mathrm{~s}$. Following a washing step, $8 \mu \mathrm{L}$ of anti-fluorescent quencher was added to the slide, and the image was captured using a fluorescence microscope as soon as possible.

\section{Statistical analysis}

The statistical data were analyzed by SPSS 19.0 statistical analysis software, and statistical graphing was performed using GraphPad Prism. The data of the two groups were compared using a t-test of independent samples. Multigroup data were compared by Kruskal-Wallis one-way analysis of variance (ANOVA). The Bonferroni method was used for comparison correction analysis. $\mathrm{P}<0.05$ was considered statistically significant.

\section{Results}

\section{Patbology of the myocardium and pericardium}

Two rats died in the CP8W group after pericardial injection, (mortality of $20 \%$ ), three rats died in the CP16W group (mortality of $30 \%$ ), and there was no death in the $\mathrm{N}$ group. The pathological observation showed the pericardium of the parietal layer in the $\mathrm{N}$ group was thin, transparent and easy to separate from the heart without adhesion. The pericardium of the visceral layer on the surface of the heart was almost invisible with the naked eye (Figure $1 A$ ). The pericardium of the CP-8W and CP-16W model groups was obviously thickened and adhered, with poor transparency, turbidity and whiteness. The pericardium of the parietal layer and the heart were difficult to separate (Figure 1B,C). Sirius red staining demonstrated that the pericardium of the visceral layer in the $\mathrm{N}$ group was a single layer and Sirius red positive (Figure 1D); the pericardium of the visceral layer in the CP-8W and CP-16W groups was significantly thickened and Sirius red positive (Figure 1E,F). Microscopic observation showed that local collagen fibers with Sirius red staining were observed in the subepicardial myocardium in the CP-8W group, while Sirius red stained collagen fibers were observed in the subepicardial, middle and endocardial myocardium in the CP-16W group (Figure $1 G-I$ ).

\section{Expression of related marker with myocardial tissue in CP} rat model

\section{The mRNA expression of a MF indicator in the CP rat model}

The mRNA expression levels of the MF indicator $\alpha$-SMA were detected in the myocardial tissue from the model and $\mathrm{N}$ groups. The results showed that the $\alpha$-SMA mRNA of the CP-8W group was higher than that of the $\mathrm{N}$ group, while the $\alpha$-SMA mRNA of the CP-16W model group was higher than that of the CP-8W model group $(\mathrm{P}<0.05$; Figure $2 A)$.

\section{Expression of miR-146a and the miR-146a target gene in the CP rat model}

The mRNA expression levels of miR-146a and its target genes, IRAK1 and TRAF6, in the CP model and $\mathrm{N}$ groups were detected in the myocardial tissue. The expression of miR-146a in CP-8W group was higher than that in the $\mathrm{N}$ group and $\mathrm{CP}-16 \mathrm{~W}$ group $(\mathrm{P}<0.05)$, but there was no significant difference between the CP-16W model group and the $\mathrm{N}$ group $(\mathrm{P}>0.05$; Figure $2 A)$. The expression levels of IRAK1 and TRAF6 in the CP-16W model group were higher than in the $\mathrm{N}$ model group and CP-8W group $(\mathrm{P}<0.05)$, while the expressions of IRAK1 and TRAF6 in the $\mathrm{CP}-8 \mathrm{~W}$ group and $\mathrm{N}$ group were not significantly different ( $>0.05 ;$ Figure 2A). Compared with the $\mathrm{N}$ group, the protein expression of $\alpha$-SMA, IRAK1, TRAF6 and p-NF$\kappa \mathrm{B}$ in the myocardial tissue of CP-8W and CP-16W groups were significantly increased $(\mathrm{P}<0.05)$, while there was no significant difference in $\mathrm{NF}-\kappa \mathrm{B}$ among the groups $(\mathrm{P}>0.05$; Figure 2B,C).

\section{miR-146a mediates the TLR-4 signaling pathway to affect MF in CFs}

\section{Primary culture and identification of rat CFs}

Neonatal rat CFs were harvested and cultured. The cells were polygonal and paved, with large nuclei. Multiple vesicles were observed in the cytoplasm, which was consistent with the morphology of rat CFs (Figure $3 A$ ). Cellular immunofluorescence staining showed vimentin expression was positive, which was consistent with rat CFs characteristics (Figure 3B).

\section{Transfection efficiency of miR-146a mimics and inhibitors}

When the rat CFs reached about $70 \%$ confluency, the miR-146a mimic (miR-146a-M) and inhibitor (miR146a-I), nonsense sequence (NC) and inhibitor nonsense 

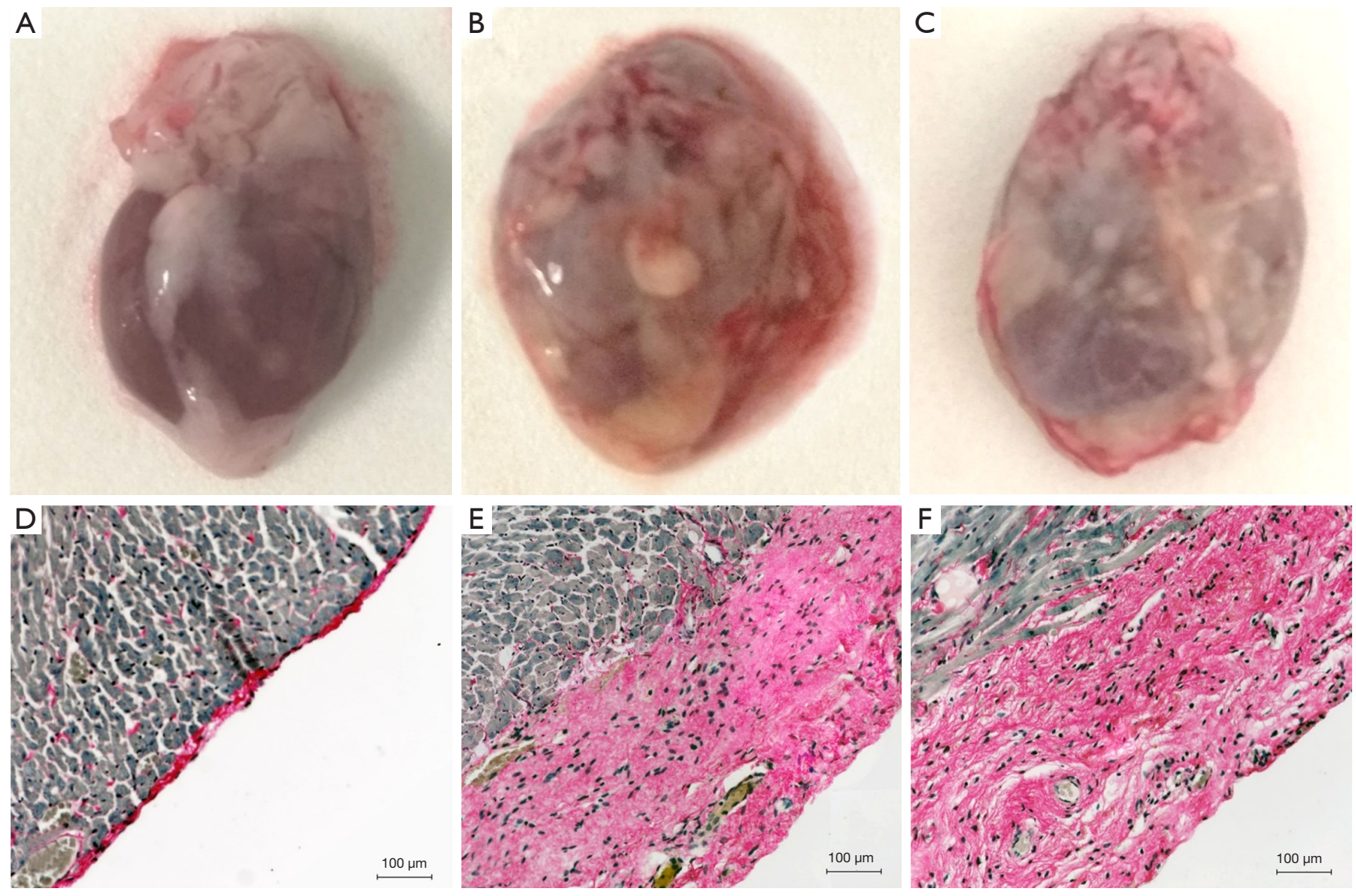

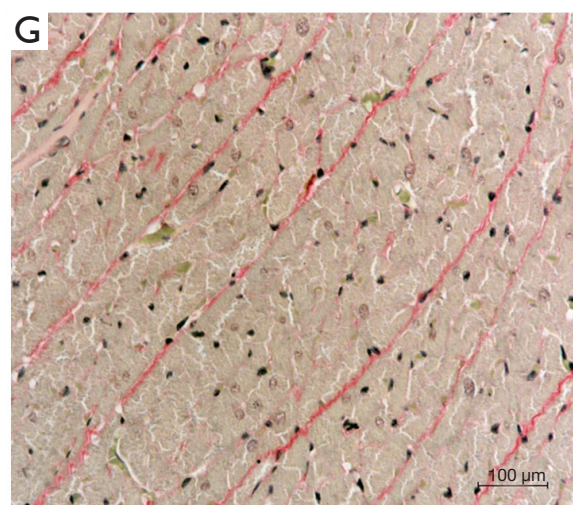

$\mathrm{N}$

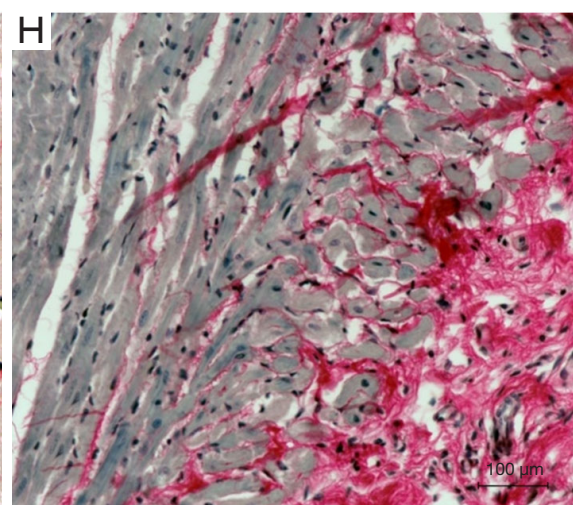

CP-8W

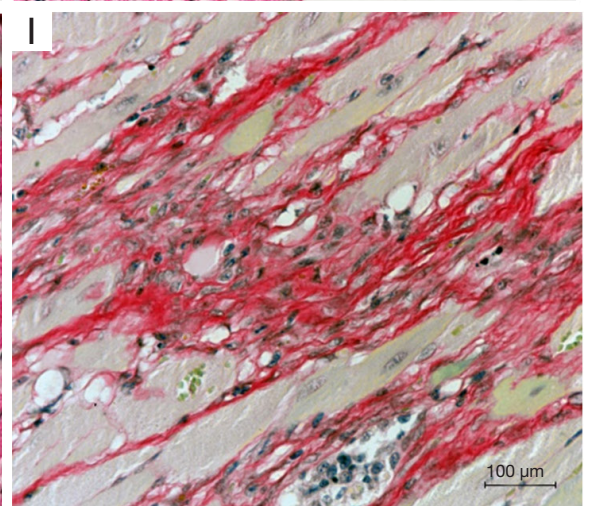

CP-16W

Figure 1 Histopathological staining of the myocardial tissue in the CP rat model. Cardiac macrospecimens of (A) the $\mathrm{N}$ group, (B) the CP-8W group, and (C) the CP-16W group; (D) Sirius red staining of the visceral layer pericardium in the N group (200x); (E) Sirius red staining of the visceral layer pericardium in the CP-8W group (200x); (F) Sirius red staining of the visceral layer pericardium in the CP16W group (200x); (G) Sirius red staining of the myocardium in the N group (200x); (H) Sirius red staining showed collagen fibers in the subepicardial myocardium of the CP-8W group (200x); (I) Sirius red staining showed collagen fibers in the endocardial myocardium of the CP-16W group (200x). 
A

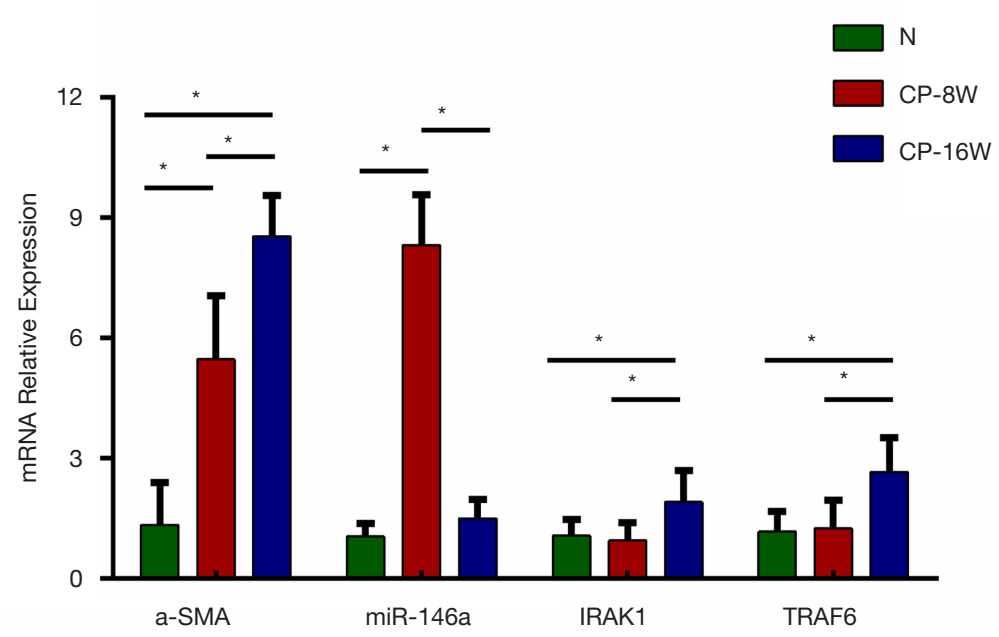

B

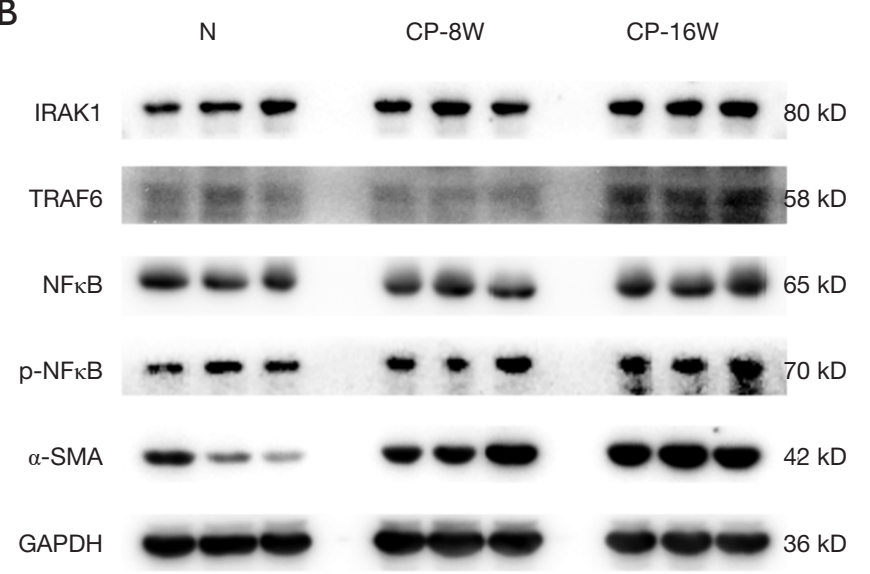

C

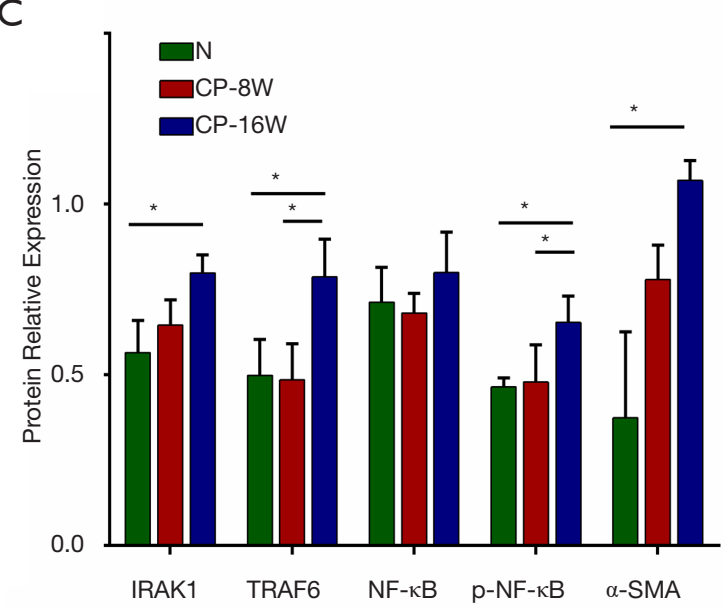

Figure 2 Expression of related marker with myocardial tissue in constrictive pericarditis (CP) rat model. (A) The mRNA expression of miR146a, IL-1 receptor-associated kinase 1 (IRAK1), tumor necrosis factor receptor-associated factor 6 (TRAF6) and $\alpha$-SMA in the CP rat

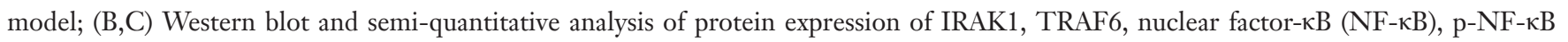
and $\alpha$-SMA in the CP rat model. GAPDH was used as a control. ${ }^{*} \mathrm{P}<0.05$.

sequence (NC-I) were transfected. The mimic increased the expression of miR-146a approximately 600 -fold, and the inhibitors reduced the expression of miR-146a by about $50 \%$, with high transfection efficiency (Figure 3C).

\section{IRAK1, TRAF6 and $\alpha$-SMA mRNA and protein} expression levels in CFs after transfection of miR-146a mimics and inhibitors

mRNA and protein expression levels of the miR-146a target genes IRAK1 and TRAF6, and the MF indicator, $\alpha$-SMA, in rat CFs were detected after transfection with miR-146a mimics or inhibitors in conjunction with LPS treatment.
Compared with the control group, there was no difference in mRNA and protein expression of IRAK1, TRAF6 and $\alpha$-SMA in the NC group or the NC-I group. Compared with the control group, mRNA and protein expression of IRAK1, TRAF 6 and $\alpha$-SMA was increased in the LPS treatment group. Compared with the only LPS treatment group, mRNA and protein expression of IRAK1, TRAF6 and $\alpha$-SMA was decreased after transfection of miR-146a mimics. In contrast, when cells were transfected with miR146a inhibitors mRNA and protein expression of IRAK1, TRAF 6 and $\alpha$-SMA was increased compared with the only LPS treatment group (Figure 4A,B). 
A

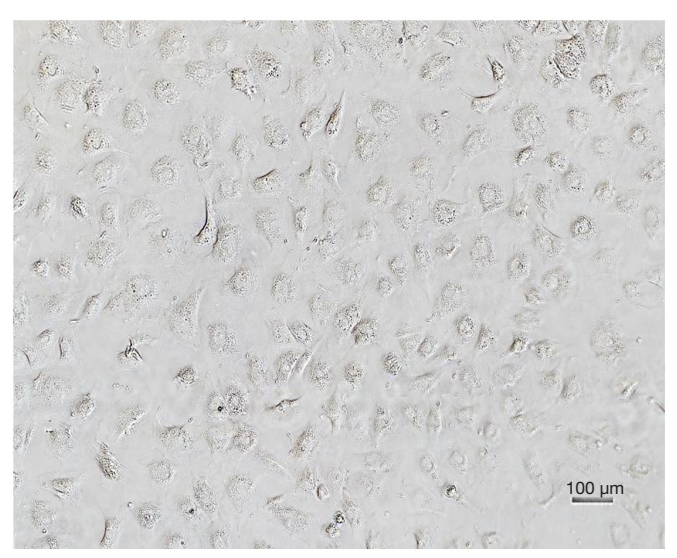

C

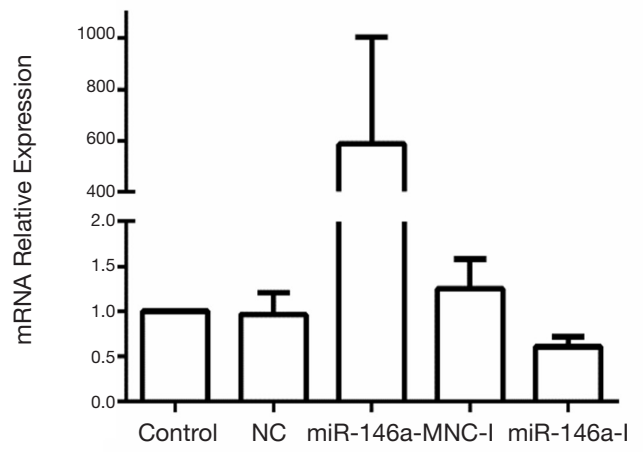

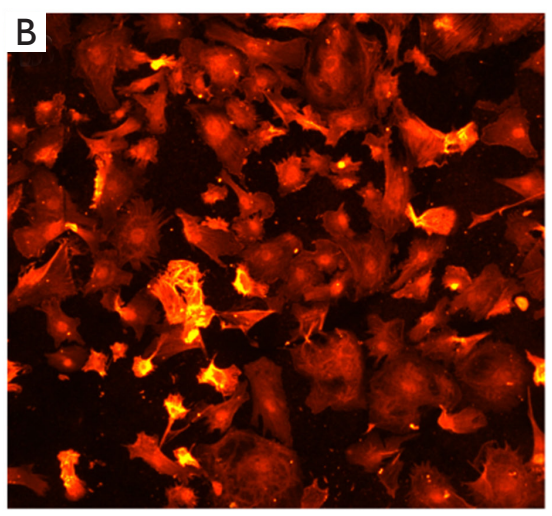

VIM

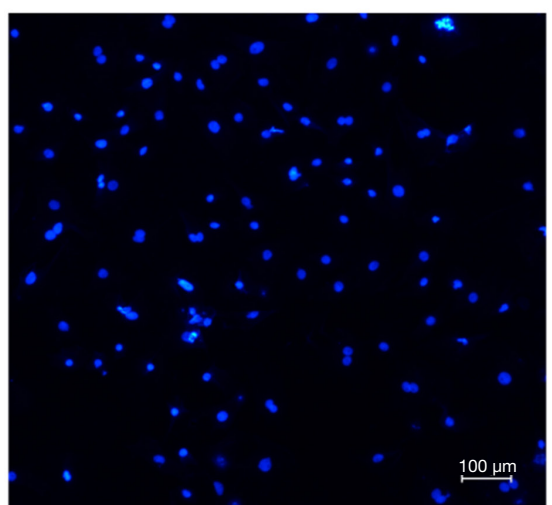

DAPI

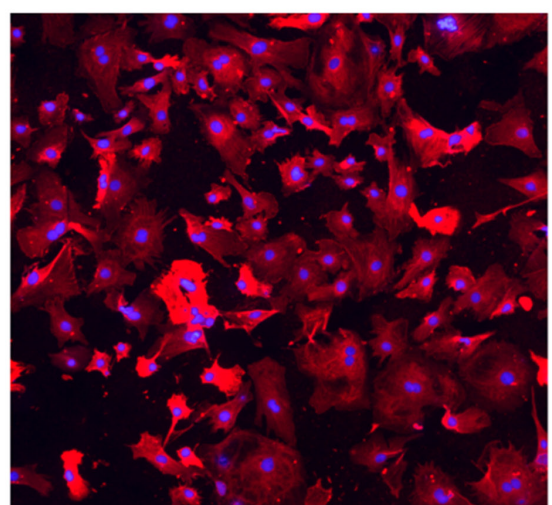

Merged

Figure 3 Transfection efficiency of miR-146a mimics and inhibitors in primary cultured rat cardiac fibroblasts (CFs). (A) Characteristics of primary cultured neonatal rat CFs; (B) identification of primary cultured neonatal rat CFs with vimentin (VIM) and 4',6-diamidino-2phenylindole (DAPI) immunofluorescence; (C) transfection efficiency of miR-146a mimics and inhibitors.

\section{Immunofluorescence detection of $\boldsymbol{\alpha}$-SMA expression}

The fluorescence intensity of $\alpha$-SMA in the LPS treatment group was higher than in the control group. After transfection of NC and NC-I, the fluorescence intensity of $\alpha$-SMA was not significantly different from that of the control group. In cells transfected with miR-146a mimics and receiving LPS treatment, the $\alpha$-SMA fluorescence intensity was lower than in the LPS group. After transfection with miR-146a inhibitors and LPS treatment, the fluorescence intensity of $\alpha$-SMA was higher than that of the LPS group (Figure 4C).

\section{Discussion}

Various pathogenic factors cause CF to proliferate and transform into myofibroblasts, which results in increased secretion of extracellular matrix and leads to MF (14). As a result, myocardial stiffness increases, compliance decreases and systolic and diastolic function declines, which, in turn, leads to a decrease in cardiac ejection capacity and, ultimately, to heart failure (15). In our study, after injection of an LPS and TP mixture through the pericardium, observation of the cardiac macrospecimens revealed that the pericardium in the $\mathrm{CP}$ group was significantly thickened and adhered, the transparency became poor, the turbidity became white, and the parietal pericardium and the heart were difficult to separate. The parietal pericardium was taken off to avoid the influence of the inflammatory reaction. The great vessels and atrium tissue were cut off to make the analysis more comparable. SR staining showed that the visceral pericardium of the CP group was significantly thickened, and local collagen fiber staining could be seen in the myocardial tissue space under the epicardium. RTPCR and Western blot detection both showed that the 
A

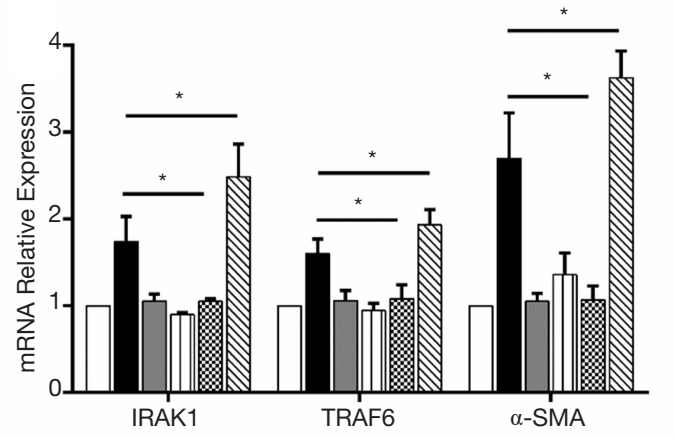

C $\alpha-S M A$
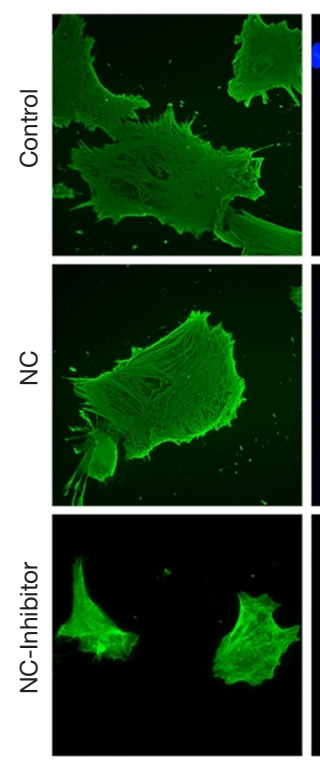

DAPI
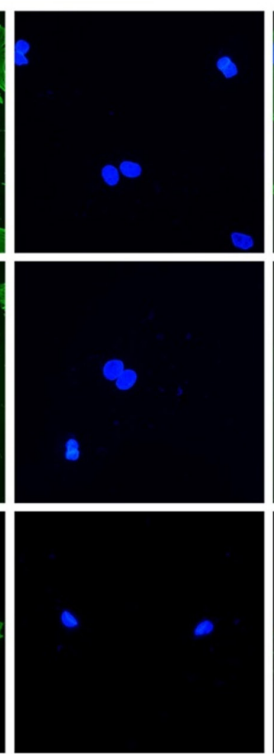

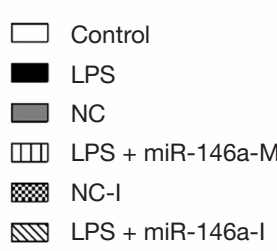

B
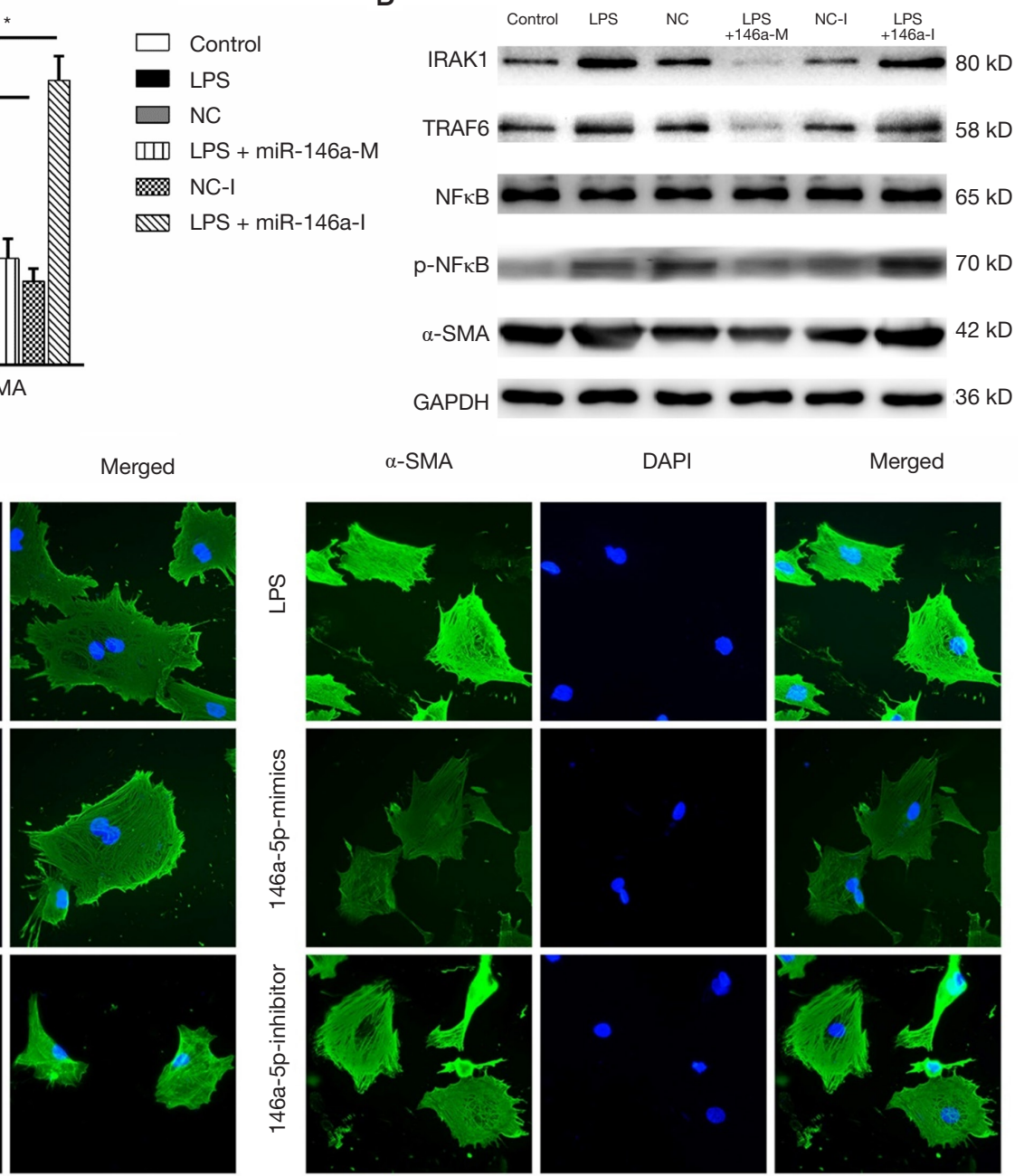
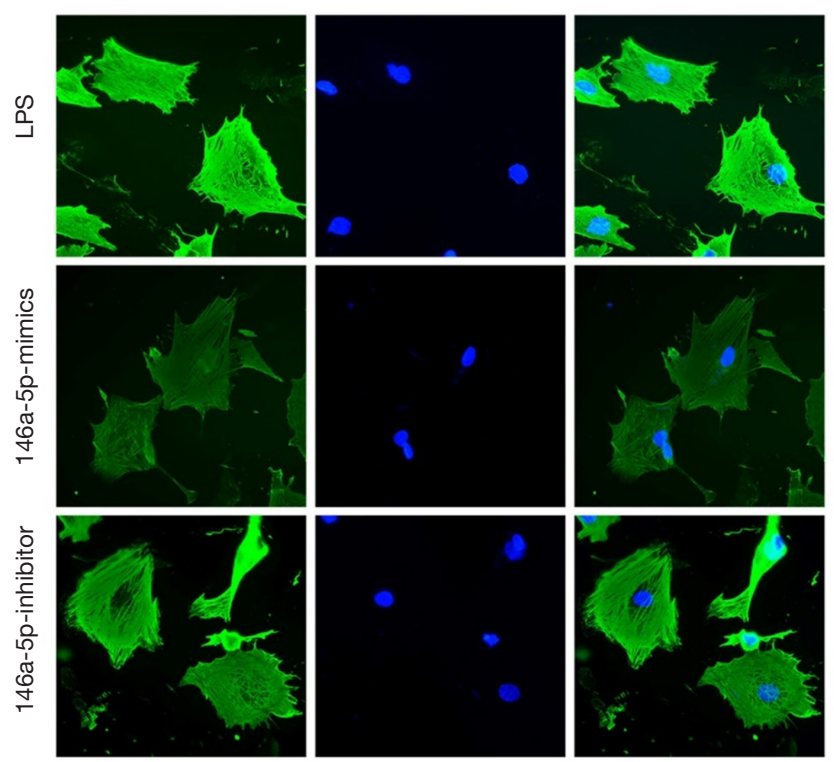

Figure 4 MiR-146a mediates the TLR-4 signaling pathway to affect MF in cardiac fibroblasts (CFs). (A,B) RT-PCR and Western blotting results of IL-1 receptor-associated kinase 1 (IRAK1), tumor necrosis factor receptor-associated factor 6 (TRAF6) and $\alpha$-SMA mRNA in CFs transfected with miR-146a mimics or inhibitors with lipopolysaccharide (LPS) treatment; (C) $\alpha$-SMA immunofluorescence in each CF group. Three independent experiments were performed. ${ }^{*} \mathrm{P}<0.05$.

expression of $\alpha$-SMA in the CP model group increased. Both macrospecimen observation and biological indicators confirmed progression of $\mathrm{MF}$ in the $\mathrm{CP}$ rats.

$\mathrm{MF}$ is considered to be associated with poor prognosis in $\mathrm{CP}(3,4)$. To date, the influencing factors and mechanisms related to MF in CP rats have not been reported. Studies have found that miRNA can play an important regulatory role in the development of MF by regulating fibroblast proliferation and differentiation, cytokine secretion, collagen metabolism and so on (16). This has broad application prospects for the diagnosis and treatment of MF. miR-146a is primarily involved in innate immune regulation, tumorigenesis and inflammatory development (7). Studies have shown that miR-146a plays an important role in tissue fibrosis, including liver and kidney fibrosis $(8,17)$. It was also found that miR-146a has a regulatory role in heart inflammation and fibrosis in diabetic patients, while the expression of miR-146a in endothelial cells and the heart was reduced in a mouse model of diabetes (18). miR146a has the ability to inhibit MMP-9 activity via the FosAP-1 pathway, which is involved in cardiac remodeling, myocardial dysfunction and heart failure (19). Wang et al. found that miR-146a can inhibit low-density lipoprotein cholesterol accumulation and the cellular inflammatory 
response by regulating the TLR-4 pathway. They also demonstrated that the expression of miR-146a is first increased and then decreased in atherosclerotic endothelial cells. The increased miR-146a has a therapeutic effect on atherosclerosis (12). The above studies indicated that the expression of miR-146a in related pathological states was mostly decreased, or increased first and then decreased. Our study found abnormally high expression of miR-146a in the 8 -week pericarditis rat model. This abnormal expression may be a promoter of MF or an early protective factor in CP.

Binding of TLR-4 to MyD88 activates the protein kinase of the IRAK family, recruits TRAF6 to form the MyD88IRAKs-TRAF6 complex, and further activates the mitogenactivated protein kinase pathway and/or NF- $\mathrm{KB}$ pathway (20). Activation of the NF- $\kappa B$ pathway is essential for MF (21). Multiple studies have shown that miR-146a is able to target IRAK1 and TRAF6 for biological effects $(22,23)$. The overall results of our study showed that the CP8W model group demonstrated no significant changes in IRAK1, TRAF6 and p-NF- $\kappa B$ compared with the control group, and the CP16W model group expressed higher IRAK1, TRAF6 and $\mathrm{p}-\mathrm{NF}-\kappa \mathrm{B}$ expression than the CP8W model group. RT-PCR showed that $\alpha$-SMA levels were higher in the CP8W model group than in the control group, and $\alpha$-SMA levels in the CP16W model group were further increased compared to the CP8W model group. These results indicated that the increased expression of miR-146a in the early stage of CP inhibits the expression of IRAK1 and TRAF6 and has an anti-cardiac fibrosis effect. However, as the disease progressed, the expression of target genes IRAK1 and TRAF6 increased, and increased NF- $\mathrm{\kappa B}$ led to negative feedback regulation, resulting in down-regulation of miR-146a and up-regulation of MF markers.

The CF cell studies also confirmed the hypothesis that upregulation of miR-146a targets TRAF6 and IRAK1 to regulate MF. We found mRNA and protein expression levels of IRAK1, TRAF 6 and the fibrosis marker $\alpha$-SMA were increased after LPS treatment in rat CFs. After transfection with miR-146a mimics, mRNA and protein expression of IRAK1, TRAF6 and fibrosis markers were decreased. After transfection with the miR-146a inhibitor, mRNA and protein expression of IRAK1, TRAF6, p-NF- $\mathrm{kB}$ and fibrosis markers increased. The fluorescence intensity of $\alpha$-SMA in the LPS treatment group was higher than it was in control group. After transfection of miR-146a mimics, the $\alpha$-SMA fluorescence intensity was lower than in the LPS group. Transfection of the miR-146a inhibitor resulted in higher $\alpha$-SMA fluorescence intensity than was seen in the LPS-only group, which is consistent with the molecular biology results. These results indicated that upregulation of miR-146a could inhibit LPS-induced fibrosis by acting on target genes IRAK1 and TRAF6, and down-regulation of miR-146a could promote fibrosis, which is consistent with the results of the study by Park et al. (24), in which miR-146a was used to regulate dendritic cell apoptosis and cytokine production by negative regulation of theTLR-4 signaling pathway.

Our research suggests there is potential for future clinical use. According to our analysis, miR-146 can be used as a biomarker to predict the course of the disease, and even as a target for treatment and drug discovery of CP patients. Although we have not conducted relevant experiments on human samples to verify due to the invasion, studies can be performed through circulation blood tests. Therefore, further researches on clinical use are feasible.

In summary, the results of gross and cellular studies confirmed that the expression of miR-146a demonstrated a dynamic change that first increased and then decreased in $\mathrm{CP}$ with different courses of MF. miR-146a may regulate MF by targeting the target genes TRAF6 and IRAK1 via the TLR-4 signaling pathway. Therefore, up-regulation of miR-146a could have a role in inhibiting MF in CP rats.

\section{Acknowledgments}

We thank International Science Editing for editing this manuscript.

Funding: This work was partially supported by the National Natural Science Foundation of China (81571686, 81701699).

\section{Footnote}

Reporting Checklist: The authors have completed the ARRIVE reporting checklist. Available at http://dx.doi. org/10.21037/jtd-20-2716

Data Sharing Statement: Available at http://dx.doi. org/10.21037/jtd-20-2716

Conflict of Interest: All authors have completed the ICMJE uniform disclosure form (available at http://dx.doi. org/10.21037/jtd-20-2716). The authors have no conflicting interests to declare.

Ethical Statement: The authors are accountable for all 
aspects of the work in ensuring that questions related to the accuracy or integrity of any part of the work are appropriately investigated and resolved. All animal experiments were approved by the Ethics Committee of Shengjing Hospital (2015PS152K). The treatment of the animals during the experiment complied with the Guidelines for Animal Ethics Treatment from the Ministry of Science and Technology.

Open Access Statement: This is an Open Access article distributed in accordance with the Creative Commons Attribution-NonCommercial-NoDerivs 4.0 International License (CC BY-NC-ND 4.0), which permits the noncommercial replication and distribution of the article with the strict proviso that no changes or edits are made and the original work is properly cited (including links to both the formal publication through the relevant DOI and the license). See: https://creativecommons.org/licenses/by-nc-nd/4.0/.

\section{References}

1. Senni M, Redfield MM, Ling LH, et al. Left ventricular systolic and diastolic function after pericardiectomy in patients with constrictive pericarditis. J Am Coll Cardiol 1999;33:1182-8.

2. Wang FF, Hsu J, Jia FW, et al. Left ventricular strain is associated with acute postoperative refractory hypotension in patients with constrictive pericarditis and preserved ejection fraction. J Thorac Dis 2018;10:4147-55.

3. Levine HD. Myocardial fibrosis in constrictive pericarditis. Electrocardiographic and pathologic observations. Circulation 1973;48:1268-81.

4. Talwar KK, Narula JP, Chopra P. Myocarditis and myocardial interstitial fibrosis in constrictive pericarditis — an extended pathological spectrum? Int J Cardiol 1990;29:241-3.

5. Abonnenc M, Nabeebaccus AA, Mayr U, et al. Extracellular matrix secretion by cardiac fibroblasts: role of microRNA-29b and microRNA-30c. Circ Res 2013; 113: 1138-47.

6. Castoldi G, Di Gioia CRT, Bombardi C, et al. MiR133a regulates collagen $1 \mathrm{~A} 1$ : potential role of miR$133 \mathrm{a}$ in myocardial fibrosis in angiotensin II-dependent hypertension. J Cell Physiol 2012;227:850-6.

7. Zhang Y, Ding S, Yang J, et al. Identification of miR146a is Associated with the Aggressiveness and Suppresses Proliferation via Targeting CDKN2A in Breast Cancer. Pathol Oncol Res 2020;26:245-51.
8. Bhatt K, Lanting LL, Jia Y, et al. Anti-Inflammatory Role of MicroRNA-146a in the Pathogenesis of Diabetic Nephropathy. J Am Soc Nephrol 2016;27:2277-88.

9. Yang Z, Peng Y, Yang S. MicroRNA-146a regulates the transformation from liver fibrosis to cirrhosis in patients with hepatitis B via interleukin-6. Exp Ther Med 2019;17:4670-6.

10. Kawai T, Akira $S$. The role of pattern-recognition receptors in innate immunity: update on Toll-like receptors. Nat Immunol 2010;11:373-84.

11. Chen X, Xie M, Liu D, et al. Downregulation of microRNA-146a inhibits ovarian granulosa cell apoptosis by simultaneously targeting interleukin-1 receptor-associated kinase and tumor necrosis factor receptor-associated factor 6 . Mol Med Rep 2015;12:5155-62.

12. Yang K, He YS, Wang XQ, et al. MiR-146a inhibits oxidized low-density lipoprotein-induced lipid accumulation and inflammatory response via targeting tolllike receptor 4. FEBS Lett 2011;585:854-60.

13. Wang X, Qiao W, Xiao Y, et al. Experimental Research on the Evaluation of Left Ventricular Function by Layered Speckle Tracking in a Constrictive Pericarditis Rat Model. J Ultrasound Med 2020;39:2219-29.

14. Schwefer M, Aschenbach R, Heidemann J, et al. Constrictive pericarditis, still a diagnostic challenge: comprehensive review of clinical management. Eur J Cardiothorac Surg 2009;36:502-10.

15. Dal-Bianco JP, Sengupta PP, Mookadam F, et al. Role of echocardiography in the diagnosis of constrictive pericarditis. J Am Soc Echocardiogr 2009;22:24-33; quiz 103-4.

16. Zhuang YS, Liao YY, Liu BY, et al. MicroRNA-27a mediates the $W n t / \beta$-catenin pathway to affect the myocardial fibrosis in rats with chronic heart failure. Cardiovasc Ther 2018. [Epub ahead of print]. doi: 10.1111/1755-5922.12468.

17. Zou Y, Cai Y, Lu D, et al. MicroRNA-146a-5p attenuates liver fibrosis by suppressing profibrogenic effects of TGFß1 and lipopolysaccharide. Cell Signal 2017;39:1-8.

18. Feng B, Chen S, Gordon AD, et al. miR-146a mediates inflammatory changes and fibrosis in the heart in diabetes. J Mol Cell Cardiol 2017;105:70-6.

19. Palomer X, Capdevila-Busquets E, Botteri G, et al. miR146a targets Fos expression in human cardiac cells. Dis Model Mech 2015;8:1081-91.

20. Compagno M, Lim WK, Grunn A, et al. Mutations of multiple genes cause deregulation of NF-kappaB in diffuse large B-cell lymphoma. Nature 2009;459:717-21.

21. Jenke A, Holzhauser L, Löbel M, et al. Adiponectin 
promotes coxsackievirus B3 myocarditis by suppression of acute anti-viral immune responses. Basic Res Cardiol 2014;109:408.

22. Wang Z, Liu F, Wei M, et al. Chronic constriction injuryinduced microRNA-146a-5p alleviates neuropathic pain through suppression of IRAK1/TRAF6 signaling pathway. J Neuroinflammation 2018;15:179.

23. An R, Feng J, Xi C, et al. miR-146a Attenuates Sepsis-

Cite this article as: Xiao Y, Qiao W, Wang X, Sun L, Ren W. MiR-146a mediates TLR-4 signaling pathway to affect myocardial fibrosis in rat constrictive pericarditis model. J Thorac Dis 2021;13(2):935-945. doi: 10.21037/jtd-20-2716
Induced Myocardial Dysfunction by Suppressing IRAK1 and TRAF6 via Targeting ErbB4 Expression. Oxid Med Cell Longev 2018;2018:7163057.

24. Park H, Huang X, Lu C, et al. MicroRNA-146a and microRNA-146b regulate human dendritic cell apoptosis and cytokine production by targeting TRAF6 and IRAK1 proteins. J Biol Chem 2015;290:2831-41. 